\title{
An Inclined Jet through a Forward Expanded Hole Ejected into Mainstream over a Concave Surface $^{*}$
}

\author{
I-Chien LEE $^{* *}$ and Ping-Hei CHEN ${ }^{* *}$ \\ ${ }^{* *}$ Department of Mechanical Engineering, National Taiwan University \\ 1, Roosevelt Road Sec. 4, Taipei, Taiwan, 10617 \\ E-mail: phchen@ntu.edu.tw
}

\begin{abstract}
This study employs a digital Particle Image Velocimetry (PIV) to obtain detailed velocity distributions and flow visualization for an inclined jet through a forward expanded hole into mainstream over a concave surface at three different blowing ratios $(\mathrm{M})$ of $0.5,1.0$, and 1.5. In this study, measurements are made in three different cases to identify the interaction between the ejected flow and the mainstream. These three cases are particles seeded in the ejected flow only, particles seeded in the mainstream only, and particles seeded in both the ejected flow and the mainstream. Measured results show that the blowing ratio can significantly affect the flow field of an ejected flow into the mainstream. From the $2 \mathrm{D}$ velocity measurement on the central plane of the injection hole at $\mathrm{M}=1.0$, measured results show that a strong interaction occurs between the ejected flow and the mainstream at the leeward side of the ejected jet. In this region, the mainstream is entrained into the center plane that produces an outward radial velocity to lift the ejected flow away from the concave wall. In this study, there is no obvious radial velocity of the jet flow to up-lift itself on the central plane. The decay of the lift-off velocity from the mainstream along the streamwise direction is a cause for reattachment of a jet flow on the concave surface.
\end{abstract}

Key words: Ejected Flow, Mainstream, Particle Image Velocimetry, Particle Concentration, Velocity Defect, Lift-off Velocity

\section{Introduction}

A jet flow into a mainstream has been extensively studied because of its applications in many industrial systems. One of its major applications is for cooling gas turbine blades from high temperature inlet gases from the combustor. Despite the complex shape of a turbine blade, some studies were performed over a simplified geometry with a normal jet into a mainstream over a flat surface. The interaction between an ejected jet and the mainstream results in a complex flow field that includes counter-rotating vortex pairs (CVP) embedded in the jet ${ }^{(1)-(4)}$, shear-layer vortex windward side of the jet ${ }^{(1)}$, the horseshoe vortex upstream of the jet ${ }^{(5)}$, and the wake region beneath the downstream of the jet near the wall surface $^{(6)}$. When observed on the cross-sectional plane, the jet with embedded CVP has a kidney shape in the mainstream. Liscinsky and $\operatorname{Truc}^{(7)}$ obtained visualized pictures of an ejected flow into the mainstream from the mean seeded particle's concentration on different 
cross-sectional planes. Their study showed that a round jet normal to the mainstream had a kidney shape at blowing ratios $(\mathrm{M})$ of $1.17,1.49$, and 2.16 . Sivadas et al. ${ }^{(8)}$ showed that a kidney-shaped CVP in the jet through flow visualization that was illuminated by a laser light sheet.

However, the flow through turbine passage experiences strong curvature effect that is not observable in flow over flat surfaces. For turbine blade cooling, the coolant is ejected into the mainstream at an angle of less than $45^{\circ}$. In prior studies, the blowing ratio usually ranges from 0.5 to 2.0 . For the jet inclined to the wall with low blowing ratios, Kadotani and Goldstein ${ }^{(9)}$ examined the effects of boundary layer thickness, Reynolds number, and freestream turbulent intensity on film cooling and velocity distribution. Thole et al. ${ }^{(10)}$ measured the effect of hole exit type on the distribution of mean velocity, turbulent intensity, and turbulent shear stress for an inclined jet with a $30^{\circ}$ angle at a blowing ratio of 1.0. This causes the mainstream being induced towards the wall surface. Accounting to the lift-off effect of jet flow on film-cooling, Goldstein and Stone ${ }^{(11)}$ presented the film-cooling performance over curved surfaces. Their study showed that the injection angle significantly affects the lift-off effect of jet flow. They also mentioned that the jet flow touches down the wall surface again when the lift-off phenomenon of the jet diminishes far downstream. For film cooling over tested surface, the lift-off phenomenon of ejected jet reduces film cooling effectiveness.

A literature review shows that few studies were performed on the effect of blowing ratio and mainstream on the lift-off of an ejected flow into a mainstream over a curved surface. This study employs a digital particle image velocimetry (DPIV) to measure detailed distributions of time-averaged velocity on the central plane of injection hole. It is required to seed tracing particles in the flow for DPIV measurements. In this study, particles are seeded in the flow in three different cases including; seeding in the mainstream only, seeding in the ejected flow only, and seeding in both the mainstream and the ejected flow. When particles are seeded in the ejected flow only, the seeded particle concentration contours of the cross-sectional plane are reported to show the counter-rotating vortex pairs that occur in the ejected flow at different blowing ratios.

\section{Experimental apparatus and operating conditions}

Figure 1(a) shows a schematic view of the experimental facility. The experiments are performed in a wind tunnel with a curved test section. On a concave surface, there is a single injection hole through which the jet is ejected into the mainstream. The detailed flow velocities in the test section are measured by a digital Particle Image Velocimetry (Dantec, FlowMap 2500) system. The set-up for DPIV measurements on the flow filed is schematically shown in Fig. 1(b). A Q-switched Nd:YAG pulsed laser (NewWave, Reasearch Minilaser) provides pulsed light sheets at a wavelength of $532 \mathrm{~nm}$ with a maximum output energy of $15 \mathrm{~mJ} /$ pulse. The particle images are recorded by a Kodak ES1.0 CCD camera with a resolution of $1008 \times 1016$ pixels and a pitch of $9 \times 9 \mu \mathrm{m}$ pixel. The camera is installed with a Nikon AF Micro $60 \mathrm{f} / 2.8 \mathrm{D}$ lens and a $532 \mathrm{~nm}$ filter lens. The light sheet optical head and the CCD camera are installed on a traversing system (Dantec 41T50, 80 pulses $/ \mathrm{mm}$ ) such that the position of the laser sheet could be precisely controlled with a spatial resolution of $12.5 \mu \mathrm{m}$.

There is a Plexiglas curved duct serving as the test section. The curved duct has a $135^{\circ}$ bend at a constant radius of $382 \mathrm{~mm}$ based on the concave surface. The jet flow is injected through a forward expanded hole with a streamwise inclined angle of $35^{\circ}$ on the concave surface. The inlet diameter of the jet hole is $8 \mathrm{~mm}$, located at an angle $(\theta)$ of $34^{\circ}$ from the onset of concave curvature. The length-to-diameter ratio of the injection hole is 2.2. A sketch of the hole geometry and a measured coordinate system is shown in Fig. 2. The 


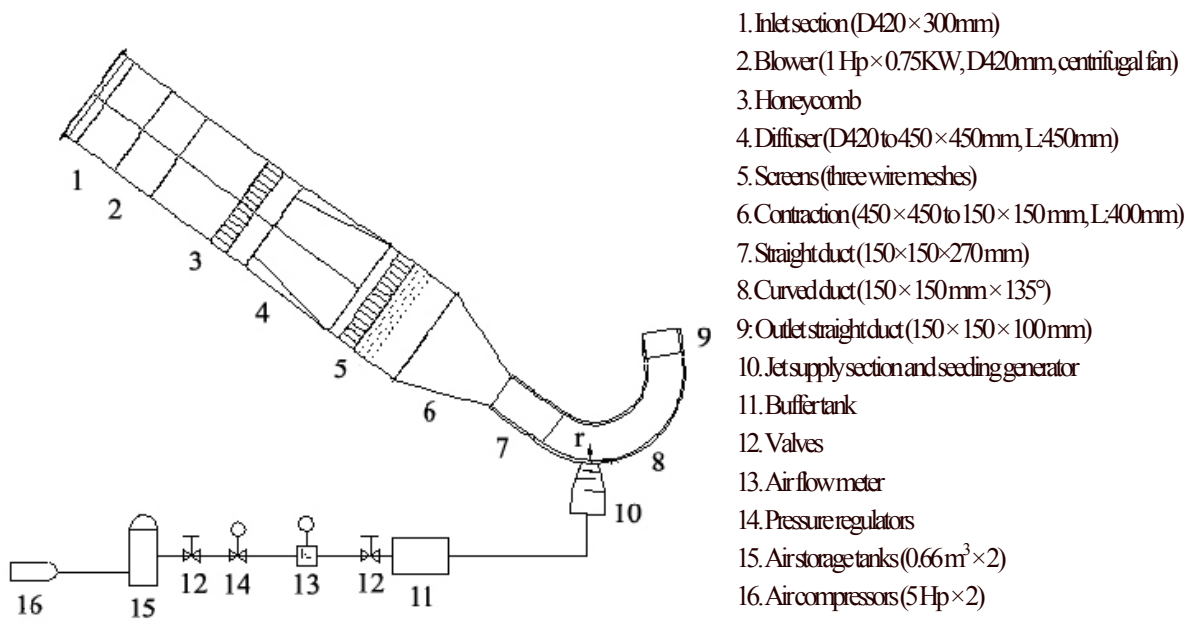

(a) The experimental set-up

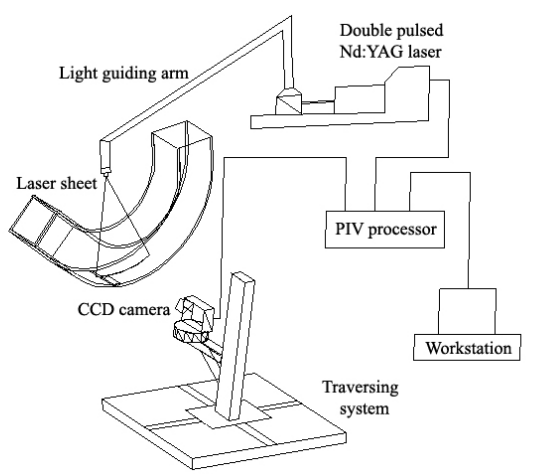

(b) The PIV system

Fig. 1 Schematic views of measurement system

mainstream velocity is also taken by a hot wire anemometer (Dantec, StreamLine 90C10) with a hot-wire probe (Dantec, 55P63) at a location $200 \mathrm{~mm}$ upstream of the onset of curved duct. A static pressure probe is installed on the concave surface. Static pressure probes are installed at different locations along a central line of the concave surface. A pressure transducer (Validyne, DP 103-16) is employed to measure the static pressure distribution along the concave surface. The measured pressure signals are converted to digital signals by an AD conversion board (Validyne, UPC 608) for further signal processing.

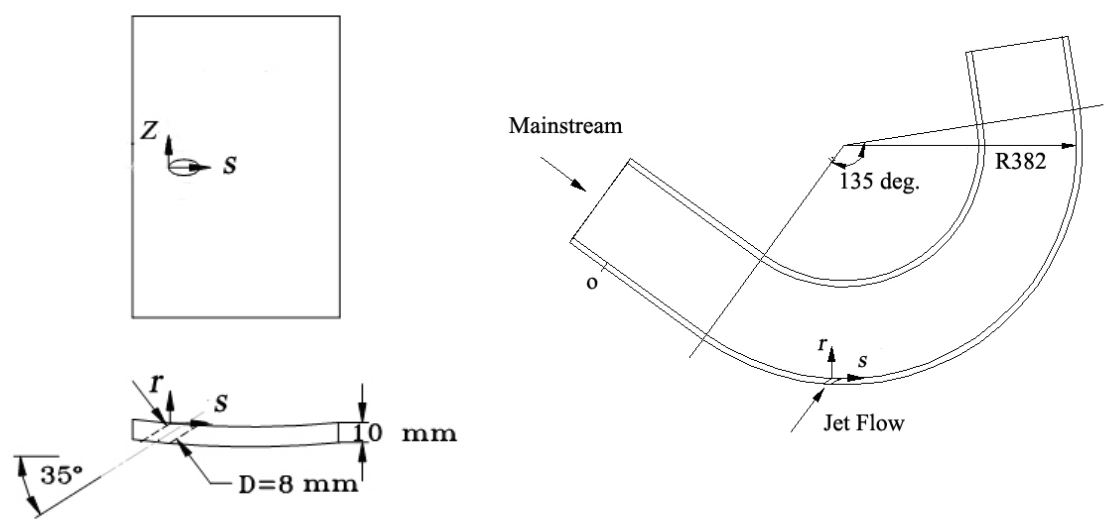

Fig. 2 Sketch of the ejected hole geometry, the curved duct and measured coordinate system 
For the PIV measurements, the velocity vectors in both streamwise and radial directions are obtained by calculating the cross-correlation coefficient between two consecutive recorded particle images. The time-averaged velocity vector distributions are acquired with a sampling rate of $15 \mathrm{~Hz}$ and are averaged over 600 instantaneous image pairs. To obtain a velocity at a specific location, the images are divided into $32 \times 32$ pixel interrogation windows with a $50 \%$ overlap grid.

The imaging process of flow visualization is the same as that in the velocity measurement. The laser light sheet is used to illuminate the cross-sectional area of the curved duct and is moved to different streamwise locations along the curved duct. The seeded particle concentration contours are acquired at a sampling rate of $15 \mathrm{~Hz}$ and are averaged over 100 instantaneous images.

During the PIV measurements, the ejected flow and the mainstream are seeded with micro-ranged particles in diameter produced by a fog generator using waterbased fog liquid ${ }^{(12)}$. If the seeding particles can move with the flow without causing any significant uncertainty, Flagan and Seinfeld ${ }^{(13)}$ showed that the particle's Stokes number, St, must be far smaller than one. In addition, the particle sedimentation velocity ${ }^{(14)}$, Ug, of seeding particles must be much smaller than the flow velocity. The particle's Stokes number and particle sedimentation velocity for the worst case in this study are 0.042 and $2.1 \times 10^{-4} \mathrm{~m} / \mathrm{s}$, respectively.

In this study, the freestream velocity of mainstream is kept at $12.0 \mathrm{~m} / \mathrm{s}$ with turbulent intensity of $1.5 \%$ at $200 \mathrm{~mm}$ upstream of the onset of the curved section. The mainstream Reynolds number is 5820 based on the diameter of the injection hole. The density ratio of the mainstream to the jet flow is 1.0 . The blowing ratios are $0.5,1.0$, and 1.5 adjusted by the mass flow rate of jet flow.

The uncertainty in the DPIV measurements can be controlled to below 4\%. Following the uncertainty calculation method proposed by Kline and $\mathrm{McClintock}^{(15)}$, average 600 images at a $95 \%$ confidence level, the uncertainty in the maximum time-averaged velocity measurements is $3.5 \%$. The uncertainties of the mainstream velocity and the mainstream turbulent intensity are $2.0 \%$ and of $2.5 \%$ respectively. The uncertainty in the coolant mass flowrate is $2 \%$. The maximum uncertainty of the pressure coefficient $\left(\mathrm{C}_{\mathrm{p}}\right)$ at a mainstream velocity of $12 \mathrm{~m} / \mathrm{s}$ is $5.6 \%$.

\section{Results and discussion}

\section{3-1 Mainstream in the curved section}

Since the freestream velocity on a concave surface of a curved duct may depend on the distance from the wall, the freestream velocity is obtained from the extrapolated velocity defined by Schultz et al. ${ }^{(16)}$ in this study. The measured boundary layer thickness at the onset of the curved section is $6.1 \mathrm{~mm}$ and it gradually increases to $17.0 \mathrm{~mm}$ at the upstream edge of the injection hole. Gillis and Johnston ${ }^{(17)}$ showed that the ratio $(\delta / \mathrm{R})$ of the boundary layer thickness at the onset of the curved section to the radius of curvature indicated the effect of curvature of the curved section on the outer layer of the mainstream. For $\delta / \mathrm{R} \leqq 0.01$, the curvature effect is generally considered to be weak to moderate; for $\delta / \mathrm{R}$ $\geqq 0.1$ it becomes significant ${ }^{(17)}$. In this study, the value of $\delta / R$, based on the concave radius of curvature, is 0.016 .

Figure 3 shows the variations of pressure coefficient $\left(C_{p}\right)$ that are recorded from the pressure taps located along the centre line of an injection hole without ejected flow on the concave surface at $\mathrm{U}_{\infty}=12 \mathrm{~m} / \mathrm{s}$. The potential velocity at wall $\left(\mathrm{U}_{\mathrm{pw}}\right)$ is also calculated from the wall static pressure by the method of Muck et al. ${ }^{(18)}$. As observed in Figure 4, the mainstream is decelerated at the start of the curved section and then keeps at a constant velocity for the angle $(\theta)$ ranging from $19^{\circ}$ to $32^{\circ}$ on the concave surface. At the angle $(\theta)$ 
from $32^{0}$ to $116^{0}$, the value of $C_{p}$ decays slightly and the mainstream is accelerated. At the end of the curved section, the value of $\mathrm{C}_{\mathrm{p}}$ decays sharply. The mainstream experiences a strong acceleration.

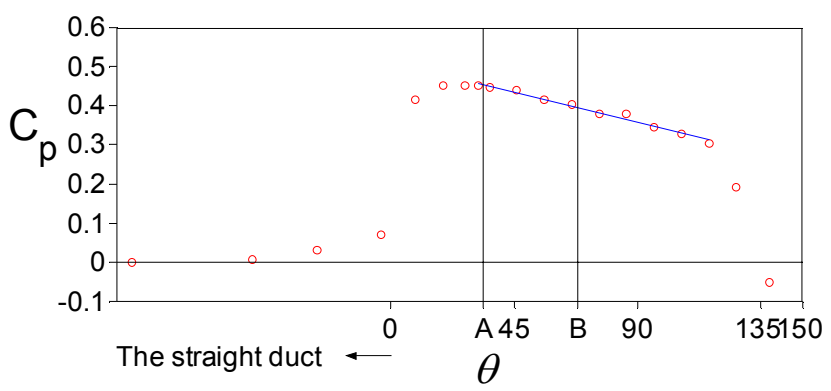

Fig. 3 Streamwise distribution of $\mathrm{C}_{\mathrm{p}}$ along the concave wall. (The DPIV measured area lies between A and B)

In this study, the pressure gradient parameter $(k)$ is $1.8 \times 10^{-9}$ and the $\mathrm{U}_{\mathrm{pw}}$ is $11.6 \mathrm{~m} / \mathrm{s}$ at the upstream edge of the injection hole. The mainstream is slightly accelerated over the concave surface in region where the PIV measurement of detailed flow field is performed for the value of $s / D$ ranging from -0.5 to 9.0 , corresponding to the angle $(\theta)$ ranging from $33.8^{0}$ to $68^{0}$.

\section{3-2 Time-averaged concentrations of a jet}

We only applied seeded particles in the ejected flow to obtain the results shown in Fig. 4 to 6 . A mixed flow zone occurs at the boundary of the ejected flow and the mainstream, diluting the seeded particle concentration in the ejected flow with the entrained mainstream which contains no seeded particles. Therefore, the value of time-averaged seeded concentration in the ejected flow, $\mathrm{C}_{\mathrm{j}}$, indicates a dominant factor of ejected flow in the mixed flow zone. A higher value of $C_{j}$ means less mainstream fluid entrained into the ejected jet at that particular location in the mixed flow zone. The ejected flow is completely dominant where $C_{j}=1.0$ in the mixed flow field. On the contrary, the mainstream is completely dominant where $\mathrm{C}_{\mathrm{j}}=0.0$ in the mixed flow field. The contours of time-averaged seeded particle concentration in the ejected flow provide a good visualization of the cross-sectional shapes of the jet flow and jet lift-off phenomenon.

One hundred instantaneous particle images are acquired through a data acquisition period of 6.67 seconds at a sampling rate of $15 \mathrm{~Hz}$. Note that the CCD images were divided into several interrogation areas $(8 \times 8$ pixels). Using the particle exist in a small interrogation area $(8 \times 8$ pixels $)$ symbolizes the jet flow pass through this measured point at that moment. When the time-averaged concentration, $\mathrm{Cj}$, equals 1.0 at the measured point, the number of pixels containing particle image is greater than 8 in the chosen interrogation area for all one hundred captured images. If the number of pixels having a bright signal is less than 8 , the bright signal is considered background noise and not a particle image.

Figures 4, 5, and 6 show the time-averaged cross-sectional concentration contours with blowing ratios $(\mathrm{M})$ of $0.5,1.0$, and 1.5 at different streamwise locations $(s / D)$ of 2.5 , 3.8, and 7.0. All three figures of contours $\mathrm{Cj}=0.9$ show that the main areas of the jet flow have clear kidney shapes, caused by a counter-rotating vortex pair ${ }^{(8)}$, at all three different blowing ratios. The contours are quite symmetric in all figures. It is quite clear that the ejected jet at low blowing ratio of 0.5 is not strong streamwise momentum enough to penetrate the mainstream. No lift-off phenomenon of ejected jet is observed at $\mathrm{M}=0.5$. Although the jet flows along the wall surface as shown in Figs. 4(b) and 4(c), the maximum time-average concentration is less than 0.82 . It reveals that the jet flow with $M$ of 0.5 is 
mixed by the mainstream on the downstream. At a medium blowing ratio of $M=1.0$, the ejected jet can lift off from the concave surface near the injection hole, shown in Fig. 5(a), but is pushed towards the concave surface by the mainstream, shown in Fig. 5(b), and finally attaches to the concave surface, shown in Fig. 5(c). At a strong blowing ratio of $M=1.5$, The strong streamwise momentum of an ejected jet can penetrate into the mainstream. The jet keeps away from the wall on the measured region. It cannot be pushed back to the concave surface by the mainstream even at a streamwise distance of $s / D=7.0$. The results above show that the jet with a smaller blowing ratio of $\mathrm{M}=0.5$ flows along the wall surface, but is mixed by a mainstream, the jet with a blowing ratio of $M=1.0$ lifts off the wall near the hole downstream and reattaches the wall surface at $s / D=3.8$, and the jet with a blowing ratio of $\mathrm{M}=1.5$ ejects away the wall on the whole measured region, For a film cooling effect of the jet ejected into a mainstream, the moderate blowing ratio of

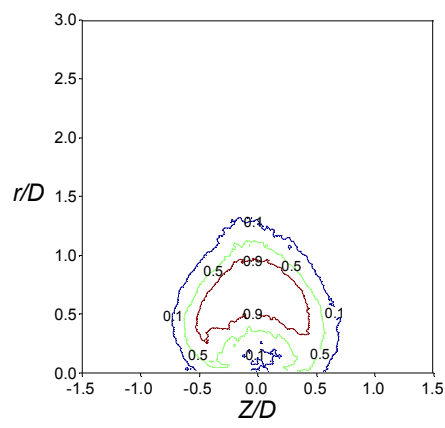

(a) $s / D=2.5$

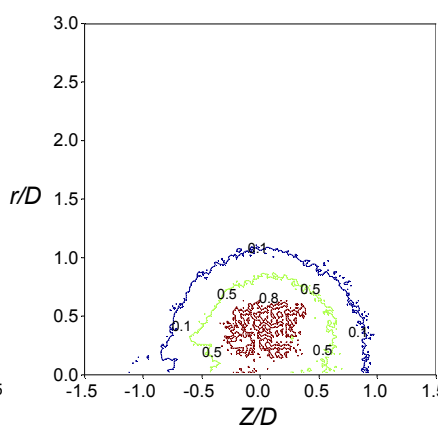

(b) $s / \mathrm{D}=3.8$

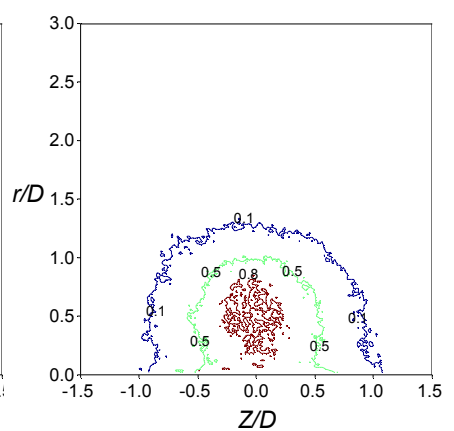

(c) $s / D=7.0$

Fig. 4 Contours of time-averaged seeded particle concentration in the ejected flow with $\mathrm{M}=0.5$

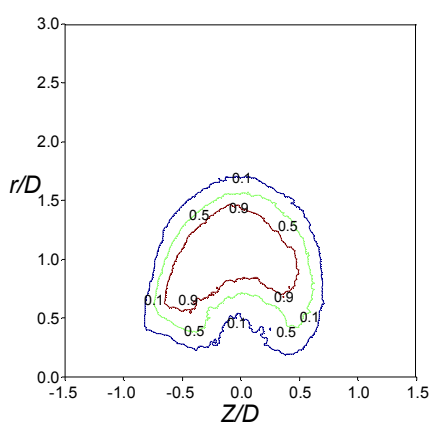

(a) $s / D=2.5$

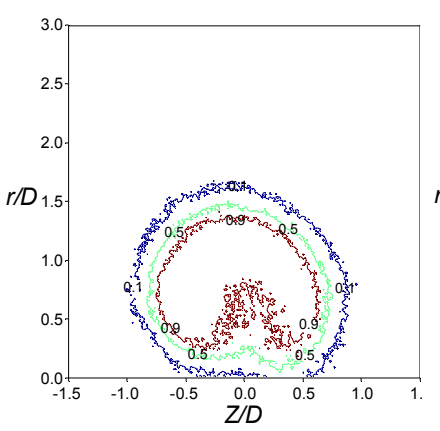

(b) $s / \mathrm{D}=3.8$

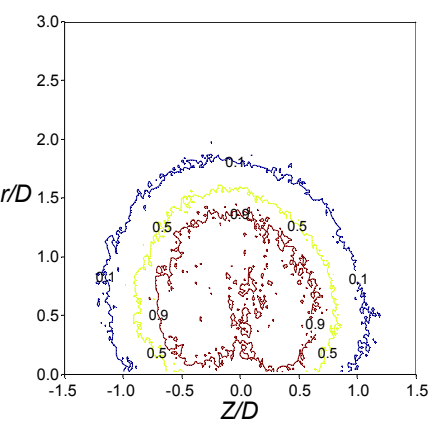

(c) $s / D=7.0$

Fig. 5 Contours of time-averaged seeded particle concentration in the ejected flow with $\mathrm{M}=1.0$

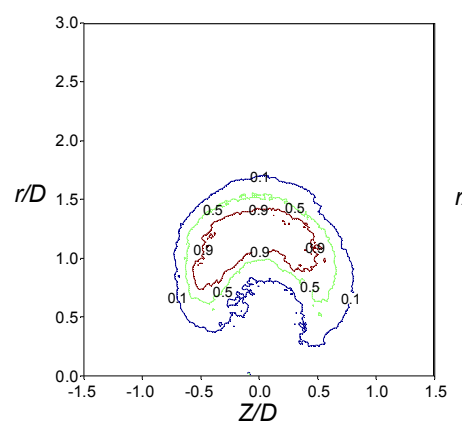

(a) $s / D=2.5$

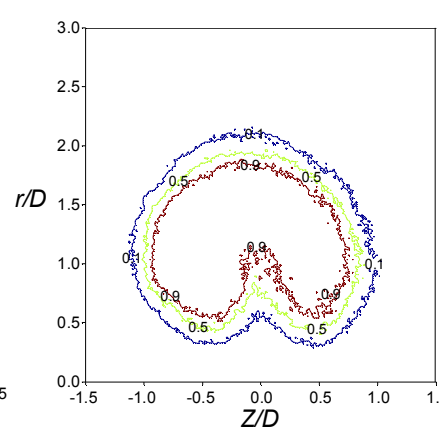

(b) $s / \mathrm{D}=3.8$

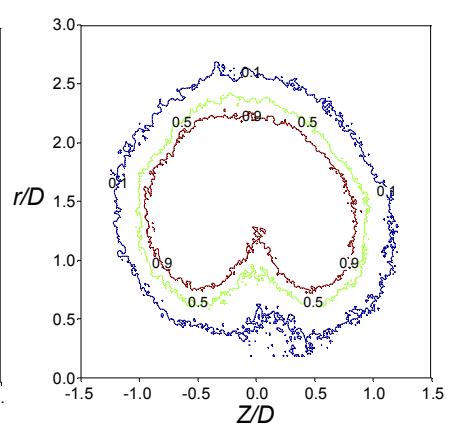

(c) $s / D=7.0$

Fig. 6 Contours of time-averaged seeded particle concentration in the ejected flow with $\mathrm{M}=1.5$ 
$M=1.0$ will give a well protection for the wall. The $2 \mathrm{D}$ time-averaged velocity field with $\mathrm{M}=1.0$ is discussed in the next section.

\section{3-3 Time-averaged velocity field}

In the PIV measurements, a pair of pictures of the CCD camera captures the reflected particle images in the flow of two consecutively pulsed light sheets. The velocity is determined from the average displacement of particles inside the interrogation over the specified time interval, and hence the extracted velocity symbolizes the velocity of an interrogation area in the measured area. The velocity field $(\mathrm{u}(r, s))$ of a mainstream with an ejected flow is measured by seeding particles in both the mainstream and the ejected flow. One can also obtain the velocity field by seeding particles in the ejected flow only. However, such a velocity field for the ejected flow in a mainstream is not appropriate particularly in regions with strong reaction between the ejected flow and the mainstream. The velocity of the ejected flow in such case, with seeding particles in the ejected flow only, are defined as a defected velocity $\left(\mathrm{u}_{\mathrm{d}}(r, s)\right)$ of the ejected flow in this study. This paper aims at presenting the time-averaged velocity contours of both real and defected velocities in the ejected flow to show a clear picture of the effect of mainstream on ejected flow. Note that all the measured results presented in this section are obtained at a blowing ratio of 1.0 on the central plane of the injection hole. The bold-faced lines in Figs. 7 to 9 are the boundaries of the ejected flow. The boundary line is defined as the interrogation area at which only 10 out of 600 images have enough particles for obtaining a valid velocity as the particles are seeded in the ejected flow only. On the leeward side of jet boundary, the reattached point of the ejected flow in mainstream is obtained at $s / D=3.4$.

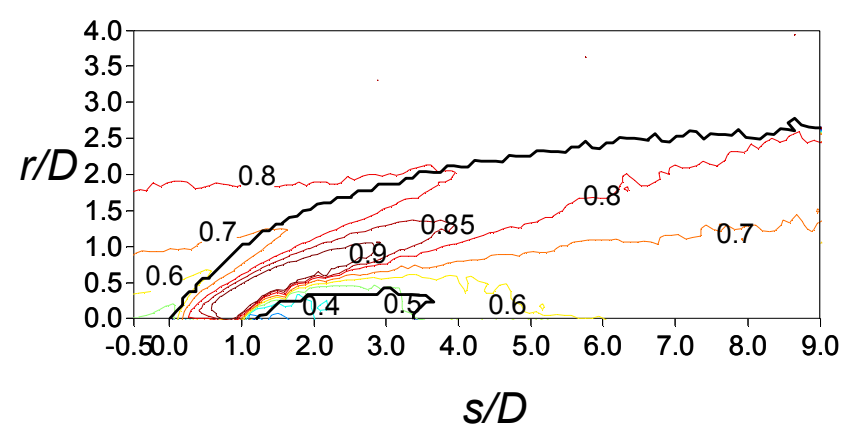

(a) $u_{s} / U_{\infty}$

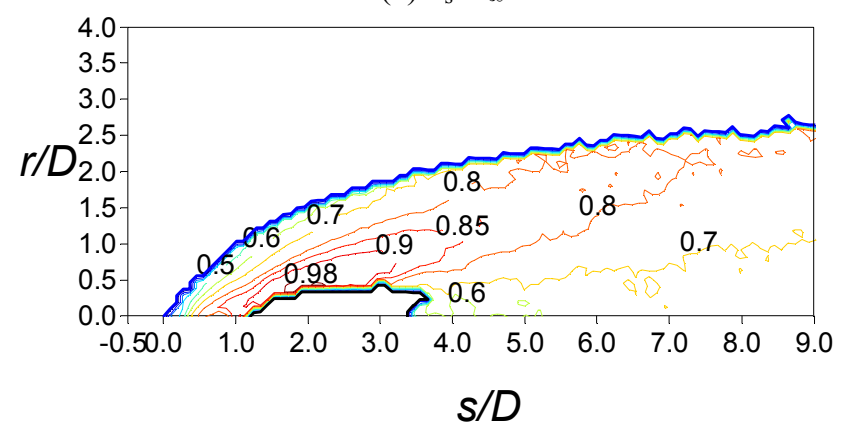

(b) $\mathrm{u}_{\mathrm{ds}} / \mathrm{U}_{\infty}$

Fig. 7 Contours of streamwise component of dimensionless velocity at $M=1.0$

Figure 7(a) and Figure 7(b) respectively show the contours of streamwise component of dimensionless time-averaged velocity $\left(\mathrm{u}_{s} / \mathrm{U}_{\infty}\right)$, and of defected velocity $\left(\mathrm{u}_{d s} / \mathrm{U}_{\infty}\right)$ of an ejected flow in a mainstream. Comparison between Fig. 7(a) and Fig. 7(b) in the jet boundary shows that both $\mathrm{u}_{\mathrm{s}}$ and $\mathrm{u}_{\mathrm{ds}}$ have similar distributions away from boundary lines. 
The value of $u_{s}$ is smaller than that of $u_{d s}$ while $u_{s}$ has a flatter velocity gradient than $u_{d s}$ in the radial direction near the leeward side of the jet boundary. It reveals that a low streamwise momentum of the mainstream gets into the jet boundary on the leeward side to mix with the ejected flow. In Fig. 7(a), the maximum value of $\mathrm{u}_{s} / \mathrm{U}_{\infty}$ is 0.91 occurring at the location $\mathrm{s} / D=1.9, r / D=0.78$. Outside the jet boundary, the value of $\mathrm{u}_{s} / \mathrm{U}_{\infty}$ symbolizes the streamwise velocity of the mainstream.

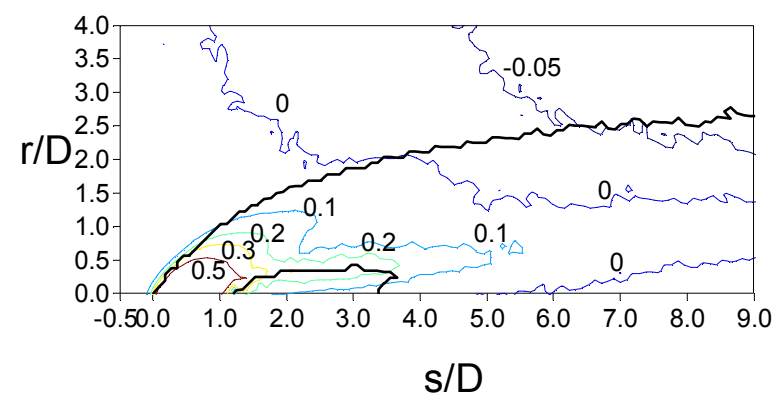

(a) $\mathrm{u}_{\mathrm{r}} / \mathrm{U}_{\infty}$

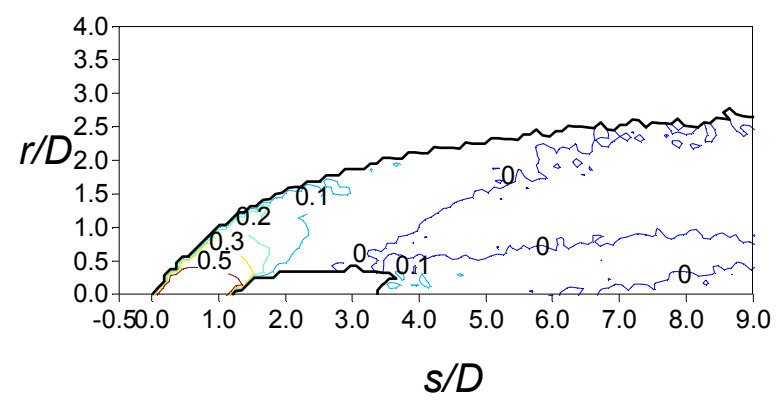

(b) $\mathrm{u}_{\mathrm{dr}} / \mathrm{U}_{\infty}$

Fig. 8 Contours of radial component of dimensionless velocity at $\mathrm{M}=1.0$

Figure 8(a) and Figure 8(b) respectively show the contours of radial component of dimensionless time-averaged velocities $\left(\mathrm{u}_{r} / \mathrm{U}_{\infty}\right)$ and of dimensionless time-averaged defected velocities $\left(\mathrm{u}_{d r} / \mathrm{U}_{\infty}\right)$ of an ejected flow in a mainstream. Comparing Fig. 8(a) and Fig. 8(b) in the jet boundary, $\mathrm{u}_{r} / \mathrm{U}_{\infty}$ has local maximum values in the radial direction from the leeward side of an ejected flow near injection hole to the downstream, but it is not so for $\mathrm{u}_{d r} / \mathrm{U}_{\infty}$. It reveals that a radial momentum of the mainstream gets into the jet boundary on the leeward side and then mixes with the ejected flow. In Fig. 8(a), there are two local maximum velocity distributions of $\mathrm{u}_{r} / \mathrm{U}_{\infty}$ along the ejected flow downstream. One mainly comes from the ejected flow starting at the injection hole, the other from the mainstream located at the leeward side of the jet boundary near the injection hole. The $\mathrm{u}_{r} / \mathrm{U}_{\infty}$, from the ejected flow, decays along the ejected flow downstream and the value $\left(\mathrm{u}_{r} / \mathrm{U}_{\infty}\right)$ is 0.1 at $s / D$ of 2.4 downstream. The $\mathrm{u}_{r} / \mathrm{U}_{\infty}$, from the mainstream, decays along the ejected flow downstream near the leeward side of the jet boundary and the value $\left(\mathrm{u}_{r} / \mathrm{U}_{\infty}\right)$ is 0.1 at $s / D$ of 5.5 downstream.

Ejecting a flow into a mainstream induces the mainstream to mix with the ejected flow near the jet boundary on the leeward side. The velocity effect of the mainstream can be determined by comparing the real flow field and the jet flow field. The $\left(u-u_{d}\right)$ can be defined as the velocity defect of the ejected flow in a mainstream. The effect of the mainstream in the jet boundary is dependent on the velocity defect. There is rarely mainstream effect in the jet boundary when the velocity defect, $\left(u-u_{d}\right)$, approaches 0 . It means that the ejected flow is rarely affected by the mainstream in this situation. The higher the magnitude of velocity defect, the more an ejected flow is mixed with the mainstream in the jet boundary. Outside 
the jet boundary, the velocity defect of the ejected flow symbolizes the velocity of the mainstream.

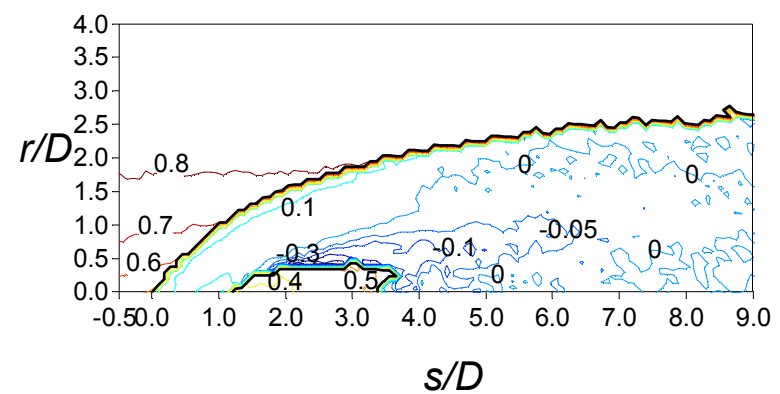

(a) the streamwise component

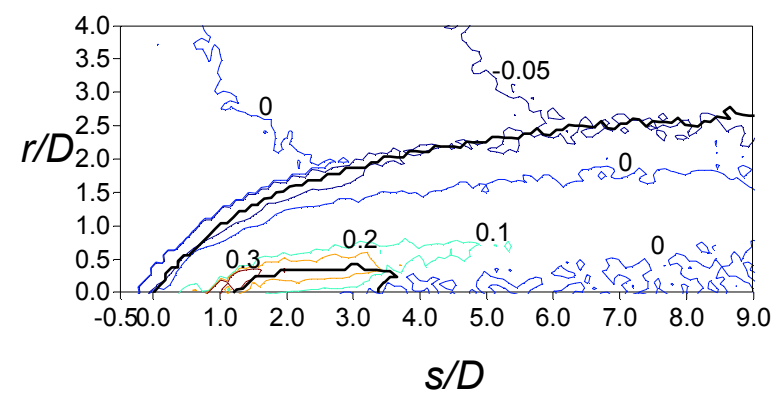

(b) the radial component

Fig. 9 Contours of dimensionless velocity defect $\left(\mathrm{u} / \mathrm{U}_{\infty}-\mathrm{u}_{\mathrm{d}} / \mathrm{U}_{\infty}\right)$ at $\mathrm{M}=1.0$

Figure 9(a) shows the contours of streamwise component of dimensionless velocity defect $\left(\left(u_{s}-u_{d s}\right) / U_{\infty}\right)$ of the ejected flow in a mainstream. In Fig. 9(a), a negative value of the velocity defect means that the velocity of the ejected flow is decreased by the mainstream in the streamwise direction located on the downside of the ejected flow. The streamwise mainstream is induced by the ejected flow towards the wall behind the injection hole and goes through the leeward side of the jet boundary. Furthermore, the streamwise mainstream depresses along the downside of the ejected flow downstream. Figure 9(b) shows the contours of radial component of dimensionless velocity defect, $\left(u_{r}-u_{r d}\right) / U_{\infty}$ of the ejected flow in a mainstream. In Fig. 9(b), a positive value of the velocity defect means that the velocity of the ejected flow is increased by the mainstream in the radial direction. Contours of the positive velocity defect along the downside of the ejected flow express that a positive radial mainstream goes through the jet boundary and mixes with the ejected flow. It reveals that the ejected flow is lifted away from the wall by the positive radial mainstream and this lift-off effect weakens as $s / D$ increases. A strong counter-rotating jet flow motion induces the mainstream to move around the jet, toward the wall, and then underneath the jet flow. This induced mainstream flow underneath the jet pushes the jet away from the wall. Lift-off velocity is defined as the positive radial velocity of induced mainstream flow underneath the jet on the hole central plane. The ejected flow is directly bent by the mainstream on the windward side. The mainstream, located on the downside of the ejected flow, possesses a lift-off velocity to keep the ejected flow away from the wall. The degradation of the lift-off effect should explain the way the jet flow touches down the wall surface again along the wall downstream. Similar effects have also been observed by means of an ammonia-diazo visualization $^{(11)}$. 


\section{Conclusions}

This study performs velocity measurements and flow visualization for a mixing flow of an inclined jet though a forward expanded hole into mainstream over a concave surface by a PIV system. The jet has a streamwise inclined angle of $35^{\circ}$ with a forward expanded angle of $8^{\circ}$. The diameter of jet hole is $8 \mathrm{~mm}$. The freestream Reynolds number of mainstream is 5820 based on the diameter of the injection hole. The PIV measurement of detailed flow field is performed for the value of $s / D$ ranging from -0.5 to 9.0 , corresponding to the angle $(\theta)$ ranging from $33.8^{0}$ to $68^{0}$. In this measured region, the mainstream without an ejected flow is slightly accelerated over the concave surface in the curved duct. The time-averaged cross-section seeded particle concentrations of an ejected flow and 2D streamwise and radial time-averaged velocity distributions on the center plane of an ejected hole have been shown. The following conclusions are obtained:

Different blowing ratios $(\mathrm{M})$ of $0.5,1.0$, and 1.5 respectively can signify that the ejected flows is in a mainstream flows along wall surface, lifts up and touches the wall surface again, and lifts farther from wall surface from measurements of cross-section seeded particle concentration.

For the velocity measurement at $\mathrm{M}=1.0$, the result shows that the jet flow and the mainstream have obvious interaction in the leeward side of the jet boundary on the central plane of an injection hole. In this region, the mainstream produces a lower streamwise velocity to dampen the velocity of an ejected flow and a radial velocity to lift the ejected flow away from the wall.

In this study, there is no obvious lift-off velocity of the ejected flow with blowing ratio of 1.0 for $s / D>2.4$ located on the central plane of an injection hole. However, the mainstream provides a lift-off velocity on the leeward side of the jet boundary and this lift-off effect weakens as $s / D$ increases.

An ejected flow in a mainstream is directly bent by the mainstream on the windward side. The mainstream, located on the downside of the ejected flow, possesses a lift-off velocity to keep the ejected flow with blowing ratio of 1.0 away from the wall. The degradation of the lift-off effect should explain the way the jet flow touches down the wall surface again along the wall downstream.

\section{Nomenclature}

$\mathrm{C}$ the time-averaged concentration

$\mathrm{C}_{\mathrm{p}} \quad$ pressure coefficient of the mainstream, $\equiv\left(P-P_{o}\right) /\left(0.5 \rho U_{p w}^{2}\right)$

$D \quad$ injection hole diameter at the inlet, $[\mathrm{m}]$

$k \quad$ pressure gradient parameter, $\equiv\left(v / U_{p w}^{2}\right)\left(d U_{p w} / d s\right)$

M blowing ratio, $\equiv \rho_{\mathrm{j}} u_{j} / \rho_{m} U_{\infty}$

$\mathrm{P} \quad$ static pressure at all, $[\mathrm{Pa}]$

$\mathrm{R}$ radius of curvature based on the concave surface, $[\mathrm{mm}]$

$\mathrm{U}_{\mathrm{pw}}$ potential velocity at wall of mainstream, $[\mathrm{m} / \mathrm{s}]$

$\mathrm{U}_{\infty}$ the rms value of freestream velocity of mainstream in the straight duct, $[\mathrm{m} / \mathrm{s}]$

u time-averaged velocity using $\mathrm{rms}$ method, $[\mathrm{m} / \mathrm{s}]$

$u_{d}$ defected velocity, velocity measured in the case with seeded particles in the ejected flow only, $[\mathrm{m} / \mathrm{s}]$

$r \quad$ radial distance from the upstream leading edge of injection hole, [mm]

$s \quad$ streamwise distance from the upstream leading edge of injection hole, $[\mathrm{mm}]$

Re Reynolds number based on the diameter of injection hole, $\equiv \rho_{m} \mathrm{U}_{\infty} D / \mu$

$\mathrm{Z}$ spanwise distance from the upstream leading edge of injection hole, $[\mathrm{mm}]$

Greek Symbols

$\beta \quad$ injection angle, [degree] 
$\theta \quad$ the angle of curved section at the onset of the bend, [degree]

$\delta \quad 0.995$ boundary layer thickness, [mm]

$\mu \quad$ dynamic viscosity of mainstream, $[\mathrm{kg} / \mathrm{ms}]$

$\rho$ density, $\left[\mathrm{kg} / \mathrm{m}^{3}\right]$

Subscripts

j jet flow

m mainstream

o reference point

$r$ radial direction

$s \quad$ streamwise direction

\section{References}

(1) Kelso, R.M., Lim, T.T., and Perry, A.E., An experimental study of round jets in cross-flow, J. Fluid Mech., Vol.306 (1996), pp.111-144.

(2) Smith, S.H., and Mungal, M.G., Mixing, structure and scaling of the jet in crossflow, J. Fluid Mech., Vol.357 (1998), pp.83-122.

(3) Cortelezzi, L., and Karagozian, A.R., On the formation of the counter-rotating vortex pair in transverse jets, J. Fluid Mech., Vol.446 (2001), pp347-373.

(4) Peterson, S.D., and Plesniak, M.W., Evolution of jets emanating from short holes into crossflow, J. Fluid Mech., Vol.503 (2004), pp.57-91.

(5) Krothapalli, A., Lourenco, L., and Buchlin, J.M., Separated flow upstream of a jet in a crossflow, AIAA J., Vol.28 (1990), pp.414-420.

(6) Fric, T.F., and Roshko, A., Vortical structure in the wake of a transverse jet, J. Fluid Mech., Vol.279 (1994), pp.1-47.

(7) Liscinsky, D.S., Truc, B., and Holdman, J.D., Crossflow mixing of noncircular jets, J. Propuls. Power, Vol.12, No.2 (1996), pp.225-230.

(8) Sivadas, V., Pani, B.S., and Butefiscb, K.A., Laser diagnostics of transverse turbulent jets, J. Flow Vis. Image Process., Vol.8 (2001), pp.369-382.

(9) Kadotani K., and Goldstein, R.J., On the nature of jets entering a turbulent flow, part a. jet-mainstream interaction, J. Eng. Power, Vol.101 (1979), pp.459-465.

(10) Thole, K., Gritsch, M., Schulz, A., and Wittig, S., Flowfield measurement for film cooling holes with expanded exits, The Int. Gas Turbine and Aero. Cong. and Exhibition, Birmingham, UK, 1996.

(11) Goldstein, R.J., and Stone, L.D., Row-of-holes film cooling of curved walls at low injection angles, J. Turbo. Trans. ASME, Vol.119 (1997), pp.574-579.

(12) Melling, A, Tracer particles and seeding for particle image velocimetry, Meas. Sci. Technol. Vol.8 (1997), pp.1406-1416.

(13) Flagan, R.C. and Seinfeld, J.H., Fundamental of air pollution engineering, Prentice Hall, Englewood Cliffs, New Jersey, (1998) pp.290-357.

(14) Raffel, M., Willert, C. and Kompenhans, J., Particle image Velocimetry: a practical guide, Springer, (1998), pp.13-16.

(15) Kline, S.J., and Mcclintock, F.A., Describing uncertainties in single sample experiments, Mechanical Engineering, (1953), pp.3-8.

(16) Schultz, M.P., and Volino, R.J., Effects of concave curvature on boundary layer transition under high freestream turbulence conditions, J. Fluids Eng. Vol.125 (2003), pp.18-27.

(17) Gillis, J.C., and Johnston, J.P., Turbulent boundary-layer flow and structure on a convex wall and its redevelopment on a flat wall, J. Fluid Mech., Vol.135 (1983), pp.123-153.

(18) Muck, K.C., Hoffmann, P.H., and Bradshaw, P., The effect of convex surface curvature on turbulent boundary layers, J. Fluid Mech. Vol.161 (1985), pp.347-369. 